TRANSACTIONS OF THE

AMERICAN MATHEMATICAL SOCIETY

Volume 361, Number 7, July 2009, Pages 3767-3791

S 0002-9947(09)04663-7

Article electronically published on February 10, 2009

\title{
ON THE GROWTH OF LOGARITHMIC DIFFERENCES, DIFFERENCE QUOTIENTS AND LOGARITHMIC DERIVATIVES OF MEROMORPHIC FUNCTIONS
}

\author{
YIK-MAN CHIANG AND SHAO-JI FENG
}

Dedicated to the eightieth birthday of Walter K. Hayman

\begin{abstract}
A crucial ingredient in the recent discovery by Ablowitz, Halburd, Herbst and Korhonen $(2000,2007)$ that a connection exists between discrete Painlevé equations and (finite order) Nevanlinna theory is an estimate of the integrated average of $\log ^{+}|f(z+1) / f(z)|$ on $|z|=r$. We obtained essentially the same estimate in our previous paper (2008) independent of Halburd et al. (2006). We continue our study in this paper by establishing complete asymptotic relations amongst the logarithmic differences, difference quotients and logarithmic derivatives for finite order meromorphic functions. In addition to the potential applications of our new estimates in integrable systems, they are also of independent interest. In particular, our findings show that there are marked differences between the growth of meromorphic functions with Nevanlinna order less than and greater than one. We have established a "difference" analogue of the classical Wiman-Valiron type estimates for meromorphic functions with order less than one, which allow us to prove that all entire solutions of linear difference equations (with polynomial coefficients) of order less than one must have positive rational order of growth. We have also established that any entire solution to a first order algebraic difference equation (with polynomial coefficients) must have a positive order of growth, which is a "difference" analogue of a classical result of Pólya.
\end{abstract}

\section{INTRODUCTION}

We first set up some notation. Let $\eta$ be a fixed, non-zero complex number, $\Delta f(z)=f(z+\eta)-f(z)$, and $\Delta^{n} f(z)=\Delta\left(\Delta^{n-1} f(z)\right)$ for each integer $n \geq 2$. In

Received by the editors January 5, 2007 and, in revised form, July 25, 2007.

2000 Mathematics Subject Classification. Primary 30D30, 30D35, 39A05; Secondary 46E25, $20 \mathrm{C} 20$.

Key words and phrases. Difference operators, Poisson-Jensen formula, Wiman-Valiron theory, integrable difference equations.

This research was supported in part by the Research Grants Council of the Hong Kong Special Administrative Region, China (HKUST6135/01P and 600806). The second author was also partially supported by the National Natural Science Foundation of China (Grant No. 10501044) and by the HKUST PDF Matching Fund.

The second author thanks the Hong Kong University of Science and Technology for its hospitality during his visit from August 2004 to March 2005.

Many main results in this paper were presented in the "Computational Methods and Function Theory" meeting held in Joensuu, Finland, June 13-17, 2005.

(C)2009 American Mathematical Society 
order to simplify our notation, we shall use the same notation $\Delta$ for both a general $\eta$ and when $\eta=1$. The context will make clear which quantity is under discussion. Equations written with the above difference operators $\Delta^{n} f(z)$ are difference equations. Let $E$ be a subset on the positive real axis. We define the logarithmic measure of $E$ to be

$$
\operatorname{lm}(E)=\int_{E \cap(1, \infty)} \frac{d r}{r}
$$

A set $E \in(1, \infty)$ is said to have finite logarithmic measure if $\operatorname{lm}(E)<\infty$. We adopt a more flexible interpretation on the Bachmann-Landau "big- $O$ " notation [38. p. 11] so that for a complex function $f(z), f(z)=O(\psi(r))$ is interpreted throughout this paper to mean that there is an $r_{0}>0$ such that $|f(z) / \psi(r)|<K$ holds for some $K>0$ and for all $r=|z|>r_{0}$. Recently, there has been a renewed interest in difference and $q$-difference equations in the complex plane $\mathbf{C}$ ([2]-6], 8]-10, 14]-18, 21, 23]-24, 26, 35, 36]), and in particular, Ablowitz, Halburd and Herbst [2] proposed to use the Nevanlinna order [19] as a detector of integrability (i.e., solvability) of non-linear second order difference equations in $\mathbf{C}$ (see [2, 15], 17, 9]; see also 33-34 and [11, pp. 261-266]). Their theory is in close spirit with the classical Painlevé ODE test in which the solutions to ordinary differential equations obtained from known integrable non-linear PDEs via similarity reduction have particularly "good" singularity structure in $\mathbf{C}$. That is, "good" singularity structure of solutions to ODEs in $\mathbf{C}$ can be regarded as a manifestation of the integrability of certain non-linear PDEs (see 1], pp. 98-100). There are also some works that focus more on the function theoretic aspects of difference operators. Halburd and Korhonen established a version of Nevanlinna theory based on difference operators [16, Bergweiler and Langley 6] considered zeros of difference operators, and Ishizaki and Yanagihara 26] developed a difference version of Wiman-Valiron theory for entire functions of small growth. Halburd and Korhonen [14] and the authors [8] studied the growth of $f(z+\eta) / f(z)$ independently. The growth of $f(z+\eta) / f(z)$ is crucial in the theory ([15]) of using the Nevanlinna order as a detector of integrability of non-linear second order difference equations. In particular, the authors obtained, in [8], that for any finite order meromorphic function $f(z)$ of order $\sigma$, then for each $\varepsilon>0$,

$$
\left|\frac{f(z+\eta)}{f(z)}\right| \leq \exp \left(r^{\sigma-1+\varepsilon}\right)
$$

holds for all $r$ outside a set of finite logarithmic measure. The result was shown to be best possible in a certain sense (see [8, e.g. 2.8]). Inequality (1.2) is in direct analogy with the classical logarithmic derivative estimate by Gundersen [12,

$$
\left|\frac{f^{\prime}(z)}{f(z)}\right| \leq|z|^{\sigma-1+\varepsilon}
$$

for all $|z|$ sufficiently large and outside a set of finite logarithmic measure, which has countless applications (see e.g. [27]). In this paper we shall establish some 
estimates that compare the growths of the logarithmic difference $\log f(z+\eta) / f(z)$, the difference quotient $\Delta f / f$ and that of $f^{\prime} / f$, and their applications to difference equations. These results are extended to higher order differences and higher order derivatives. In addition, we shall also exhibit examples of meromorphic functions showing that our estimates are best possible when interpreted in an appropriate sense.

We shall establish, as our first main result, that for any finite order meromorphic function of order $\sigma$, then for any given $\varepsilon>0$,

$$
2 \pi i n_{z, \eta}+\log \frac{f(z+\eta)}{f(z)}=\eta \frac{f^{\prime}(z)}{f(z)}+O\left(r^{\beta+\varepsilon}\right),
$$

or its equivalent form

$$
\frac{f(z+\eta)}{f(z)}=e^{\eta \frac{f^{\prime}(z)}{f(z)}+O\left(r^{\beta+\varepsilon}\right)}
$$

holds outside a set of $|z|=r$ of finite logarithmic measure, $n_{z, \eta}$ is an integer that depends on both $z$ and $\eta, \beta<\lambda-1$ when $\lambda<1$ and $\beta \leq \lambda-1$ when $\lambda \geq 1$, where $\lambda$ is the exponent of convergence of the zeros and poles of $f$ (Theorem 2.1). The above result holds for all finite order meromorphic functions. In particular, it is easy to see that inequality (1.2) ([8) mentioned above follows easily from (1.4).

Our second main result shows that if the order $\sigma$ is less than one, then one can "remove" the exponential function on the right side of (1.4). More precisely, we have

$$
\frac{\Delta^{k} f(z)}{f(z)}=\eta^{k} \frac{f^{(k)}(z)}{f(z)}+O\left(r^{(k+1)(\sigma-1)+\varepsilon}\right),
$$

which holds outside a set of $|z|=r$ of finite logarithmic measure. Equation (1.5) is a consequence of the repeated applications of itself when $k=1$ and the following estimate:

$$
\left|\frac{\Delta^{k} f(z)}{f(z)}\right| \leq|z|^{k \sigma-k+\varepsilon}
$$

which again holds for any $\varepsilon>0$ and for $z$ outside a set of finite logarithmic measure. We note that our (1.6) is in direct analogy with Gundersen's (1.3). Our method of proof depends heavily on the Poisson-Jensen formula.

If we assume that $f(z)$ is an entire function, then we can establish, as a direct consequence of (1.5), a difference Wiman-Valiron estimate

$$
\frac{\Delta^{k} f(z)}{f(z)}=\eta^{k}\left(\frac{\nu(r, f)}{z}\right)^{k}+O\left(r^{k \sigma-k-\gamma+\varepsilon}\right)
$$

which holds again outside an exceptional set of finite logarithmic measure, and where $\gamma$ is a small positive constant which will be made clear later. The notation $\nu(r, f)$ in (1.7) denotes the usual central index (see s6, 39] and 32]) of $f(z)$. Although the remainder of our (1.7) differs from that of the classical estimate that involves the derivatives instead of the differences of $f(z)$, it is sufficient for most of our applications to difference equations in 97 . 
Ishizaki and Yanagihara [26] established a "difference" version of Wiman-Valiron theory by expanding the entire function not in terms of the usual basis $\left\{z^{n}\right\}$, but in terms of factorial series, that is, series written in terms of the basis $\left\{(z)_{n}\right\}$ (where $\left.(z)_{n}=z(z+1) \cdots(z+k-1)\right)$. In particular, their results are stated in terms of the central index $\nu^{*}(r, f)$ with respect to $\left\{(z)_{n}\right\}$ instead of the usual central index $\nu(r, f)$. Thus they need to impose a strong restriction that $f(z)$ has order strictly less than $1 / 2$. Since our assumption of $f$ only requires $\sigma(f)<1$, so our results have a greater flexibility. When the entire function is of order larger than one, the relation (1.7) can no longer hold since one has the relation (1.4) instead. Thus the order assumption of our result is best possible. Our (1.7) has an added advantage that it only involves the usual central index $\nu(r, f)$ instead of the $\nu^{*}(r, f)$ which could be more difficult to calculate.

Bergweiler and Langley investigated the zero distribution of $\Delta^{k} f(z)$ for entire functions of order $\sigma(f)<1$ in [6]. They obtain the relation [6, Lemma 4.2]

$$
\Delta^{k} f(z) \sim f^{(k)}(z),
$$

outside some exceptional set. Although in close scrutiny one could derive the error estimate as in our (1.5) from some of their estimates, no explicit error bound is given in their paper 1 In fact, it is clear that both the objectives and methods of approach between Bergweiler and Langley's paper and ours are very different. In addition, we exhibit examples showing that the major estimates in this paper, including equation (1.5), are the best possible in some sense (see Example 4.2). Moreover, our "logarithmic differences" approach is natural from the Nevanlinna value distribution theory viewpoint where the corresponding error bounds are also crucial in our applications to difference equations as will be discussed in the next paragraph.

Both of the estimates (1.6) and (1.7) allow us to estimate the growth of solutions in $\mathbf{C}$ of linear difference equations,

$$
P_{n}(z) \Delta^{n} f(z)+\cdots+P_{1}(z) \Delta f(z)+P_{0}(z) f(z)=0,
$$

where $P_{j}, j=0, \cdots, n$ are polynomials, as in the classical case of linear differential equations with polynomial coefficients (see 39], Chap. IV). In particular, we show that if the orders of growth of entire solutions to (1.9) are strictly less than one, then they are equal to a set of positive rational numbers, which can be obtained from the gradients of the Newton-Puisseux diagram of the associated algebraic equations. Besides, we shall also show that any entire solution $f$ to the first order algebraic difference equation

$$
\Omega(z, f(z), \Delta f(z))=0
$$

with polynomial coefficients must have a positive order of growth. This result is in close analogy with a corresponding result of Pólya 31] when $\Delta f$ is replaced by $f^{\prime}$ and with the same conclusion.

\footnotetext{
${ }^{1}$ When J. K. Langley showed us their manuscript of [6] after our presentation during the CMFT meeting in June, 2005, as noted in a footnote on our page 1, we had already obtained the main results presented in this paper.
} 
This paper is organized as follows. The main result on logarithmic differences will be stated in $\$ 2$ and proved in $\$ 3$. The result for first order difference and those of higher order differences will be stated and proved in $\$ 4$ and $\$ 5$ respectively. In addition, examples will be constructed in both of 92 and 84 showing that the corresponding main results there are best possible in a certain sense. We shall formulate difference Wiman-Valiron type estimates in \$6. Applications of our main results to linear and first order algebraic difference equations will be discussed in 87. Finally, we shall discuss different aspects of the main results obtained in this paper in 8 .

\section{A LOGARITHMiC DIFFERENCE RESUlt}

Theorem 2.1. Let $f(z)$ be a meromorphic function of order $\sigma=\sigma(f)<\infty$, and let $\lambda^{\prime}$ and $\lambda^{\prime \prime}$ be, respectively, the exponent of convergence of the zeros and poles of $f$. Then for any given $\varepsilon>0$, there exists a set $E \subset(1, \infty)$ of $|z|=r$ of finite logarithmic measure, so that

$$
2 \pi i n_{z, \eta}+\log \frac{f(z+\eta)}{f(z)}=\eta \frac{f^{\prime}(z)}{f(z)}+O\left(r^{\beta+\varepsilon}\right),
$$

or equivalently,

$$
\frac{f(z+\eta)}{f(z)}=e^{\eta \frac{f^{\prime}(z)}{f(z)}+O\left(r^{\beta+\varepsilon}\right)}
$$

holds for $r \notin E \cup[0,1]$, where $n_{z, \eta}$ in (2.1) is an integer depending on both $z$ and $\eta, \beta=\max \{\sigma-2,2 \lambda-2\}$ if $\lambda<1$ and $\beta=\max \{\sigma-2, \lambda-1\}$ if $\lambda \geq 1$ and $\lambda=\max \left\{\lambda^{\prime}, \lambda^{\prime \prime}\right\}$.

Remark 2.1. Since $2 \sigma-2<\sigma-1$ when $\sigma<1$ and $\max \{\sigma-2, \lambda-1\} \leq \sigma-1$ when $\sigma \geq 1$, so we can easily deduce the estimate (1.2) obtained in [8] as a consequence of our Theorem 2.1.

We shall construct an example here showing that the remainder in (2.2) is best possible when $\lambda=\max \left\{\lambda^{\prime}, \lambda^{\prime \prime}\right\}<1$.

Example 2.2. Let $\lambda<1$ be a positive number and define

$$
f(z)=e^{z^{k}} \prod_{n=1}^{\infty}\left(1-\frac{z}{n^{1 / \lambda}}\right) .
$$

If $\lambda=0$, then we choose $f(z)=e^{z^{k}}$. The function $f$ has order $k$ when $k \geq 1$, and it has order $\lambda$ when $k=0$, and the exponent of convergence of the zeros of $f$ is $\lambda$.

Let

$$
F=\bigcup_{n=1}^{\infty}\left[n^{\frac{1}{\lambda}}+\frac{1}{3} n^{\frac{1}{\lambda}-1}, n^{\frac{1}{\lambda}}+\frac{2}{3} n^{\frac{1}{\lambda}-1}\right] .
$$

It can be seen that $F$ has infinite logarithmic measure on the positive real axis. Let us consider $x \in F$. Then there exists an integer $m$ such that

$$
m^{\frac{1}{\lambda}}+\frac{1}{3} m^{\frac{1}{\lambda}-1} \leq x \leq m^{\frac{1}{\lambda}}+\frac{2}{3} m^{\frac{1}{\lambda}-1} .
$$


We have

$$
\begin{aligned}
& -3 m^{1-\frac{1}{\lambda}} \leq \frac{1}{m^{\frac{1}{\lambda}}-x} \leq 0 \\
& 0 \leq \frac{1}{(m+1)^{\frac{1}{\lambda}}-x} \leq 3 m^{1-\frac{1}{\lambda}}
\end{aligned}
$$

and

$$
m \geq\left(\frac{x}{2}\right)^{\lambda} .
$$

One can find positive constants $\delta$ and $c$ so that

$$
\log (1+w)-w \geq c w^{2}
$$

holds for all $w \in[-\delta, \delta]$. We consider $x \in F$ large enough such that $3 m^{1-\frac{1}{\lambda}}<\delta$. Then it follows from (2.6) and (2.7) that

$$
-\delta \leq \frac{1}{n^{\frac{1}{\lambda}}-x} \leq \delta
$$

holds for all $n \in \mathbf{N}$. We now apply (2.9) and (2.8) to get for $x$ large enough and in $F$,

$$
\begin{aligned}
\log \frac{f(x+1)}{f(x)}-\frac{f^{\prime}(x)}{f(x)}= & (x+1)^{k}-x^{k}-k x^{k-1} \\
& +\sum_{n=1}^{\infty} \log \left(1-\frac{1}{n^{\frac{1}{\lambda}}-x}\right)+\frac{1}{n^{\frac{1}{\lambda}}-x} \\
& \geq \frac{k(k-1)}{2} x^{k-2}(1+o(1))+c \sum_{n=1}^{\infty} \frac{1}{\left(n^{\frac{1}{\lambda}}-x\right)^{2}} \\
& \geq \frac{k(k-1)}{2} x^{k-2}(1+o(1))+c \frac{1}{\left(m^{\frac{1}{\lambda}}-x\right)^{2}} \\
& \geq \frac{k(k-1)}{2} x^{k-2}(1+o(1))+\frac{9}{4} c m^{2-\frac{2}{\lambda}} \\
& \geq \frac{k(k-1)}{2} x^{k-2}(1+o(1))+\frac{9}{4} c 2^{2-2 \lambda} x^{2 \lambda-2} .
\end{aligned}
$$

We can see from the above example that Theorem 2.1 is best possible when $\lambda<1$, in the sense that the exponents $\sigma-2=k-2$ and $2 \lambda-2$ in (2.1) are attained, and hence they cannot be improved.

\section{Proof of Theorem 2.1}

\subsection{Preliminary results.}

Lemma 3.1. Let us define

$$
\log w=\log |w|+i \arg w, \quad-\pi \leq \arg w<\pi
$$


to be the principal branch of the logarithmic function in the complex plane. Then we have

$$
\log (1+w)=O(|w|)
$$

and

$$
\log (1+w)-w=O\left(|w|^{2}\right),
$$

for $|w+1| \geq 1$.

Proof. We shall omit their elementary proofs.

Remark 3.1. We note that it is clear that (3.3) remains valid when we define the principal branch by $\log w=\log |w|+i \arg w,-\pi<\arg w \leq \pi$ instead.

We shall apply the above lemma to prove

Lemma 3.2. Let us assume that we choose the principal branch as in Lemma 3.1. Then we have

$$
\log (1+w)=O(|w|)+O\left(\left|\frac{w}{1+w}\right|\right)
$$

and

$$
\log (1+w)-w=O\left(|w|^{2}\right)+O\left(\left|\frac{w}{1+w}\right|^{2}\right)
$$

hold for all $w$ in $\mathbf{C}$.

Proof. If $|w+1| \geq 1$, then (3.4) easily follows from (3.2). If, however, $|w+1|<1$, then

$$
\left|1+\frac{-w}{1+w}\right|=\frac{1}{|1+w|}>1
$$

Thus we have

$$
\log (1+w)=-\log \frac{1}{1+w}=O\left(\left|1-\frac{1}{1+w}\right|\right)=O\left(\left|\frac{w}{1+w}\right|\right) .
$$

This proves (3.4) for all $w \in \mathbf{C}$.

Similarly, if $|w+1| \geq 1$ we easily see that (3.5) follows from (3.3). On the other hand, if $|w+1|<1$, then (2.9) holds, and (3.3) implies that

$$
\begin{aligned}
\log (1+w)-\frac{w}{1+w} & =-\left[\log \left(1+\frac{-w}{1+w}\right)-\left(\frac{-w}{1+w}\right)\right] \\
& =O\left(\left|\frac{w}{1+w}\right|^{2}\right) .
\end{aligned}
$$

We note that we have used a slightly different definition of the principal logarithm as mentioned in Remark 3.1 in order to handle the right hand sides of (3.7) and (3.8) above.

On the other hand, we have

$$
\begin{aligned}
\left(\frac{w}{1+w}\right)-w & =\frac{-w^{2}}{(1+w)}=-w \cdot \frac{w}{1+w} \\
& =O\left(|w|^{2}\right)+O\left(\left|\frac{w}{1+w}\right|^{2}\right) .
\end{aligned}
$$


Thus we obtain, from combining (3.8) and (3.9),

$$
\begin{aligned}
\log (1+w)-w & =\log (1+w)+\left[-\left(\frac{w}{1+w}\right)+\left(\frac{w}{1+w}\right)-w\right] \\
& =O\left(|w|^{2}\right)+O\left(\left|\frac{w}{1+w}\right|^{2}\right)
\end{aligned}
$$

which holds for all $w \in \mathbf{C}$.

Lemma 3.3 ([30, p. 163). Let $f(z)$ be a meromorphic function in the complex plane, not identically zero. Let $\left(a_{\nu}\right)_{\nu \in N}$ and $\left(b_{\mu}\right)_{\mu \in N}$, be the sequence of zeros and poles, with due account of multiplicity, of $f(z)$ respectively. Then for $|z|<R<\infty$,

$$
\begin{gathered}
\log f(z)=\frac{1}{2 \pi} \int_{0}^{2 \pi} \log \left|f\left(R e^{i \phi}\right)\right| \frac{R e^{i \phi}+z}{R e^{i \phi}-z} d \phi-\sum_{\left|a_{\nu}\right|<R} \log \frac{R^{2}-\bar{a}_{\nu} z}{R\left(z-a_{\nu}\right)} \\
+\sum_{\left|b_{\mu}\right|<R} \log \frac{R^{2}-\bar{b}_{\mu} z}{R\left(z-b_{\mu}\right)}+i C,
\end{gathered}
$$

where

$$
C=\arg f(0)-\sum_{\left|b_{\mu}\right|<R} \arg \left(-\frac{R}{b_{\mu}}\right)+\sum_{\left|a_{\nu}\right|<R} \arg \left(-\frac{R}{a_{\nu}}\right)+2 m_{z} \pi .
$$

Remark 3.2. We recover the classical Poisson-Jensen formula by taking the real parts on both sides of (3.10). Note that $m_{z} \in \mathbf{N}$ in (3.11) depends on the choice of branch of the logarithm functions of both sides of (3.10), and so it may depend on $z$ (but being piecewise continuous).

We also require the following classical Cartan lemma.

Lemma 3.4 ([7]; see also [29]). Let $z_{1}, z_{2}, \cdots, z_{p}$ be any finite collection of complex numbers, and let $B>0$ be any given positive number. Then there exists a finite collection of closed disks $D_{1}, D_{2}, \cdots, D_{q}$ with corresponding radii $r_{1}, r_{2}, \cdots, r_{q}$ that satisfy

$$
r_{1}+r_{2}+\cdots+r_{q}=2 B
$$

such that if $z \notin D_{j}$ for $j=1,2, \cdots, q$, then there is a permutation of the points $z_{1}, z_{2}, \cdots, z_{p}$, say, $\hat{z}_{1}, \hat{z}_{2}, \cdots, \hat{z}_{p}$, that satisfies

$$
\left|z-\hat{z}_{l}\right|>B \frac{l}{p}, \quad l=1,2, \cdots, p,
$$

where the permutation may depend on $z$.

\subsection{Proof of Theorem 2.1.}

Proof. For $\eta \neq 0$, let $R>|z|+|\eta|$. Then we have by (3.10) and (3.11) that

$$
\begin{aligned}
\log f(z+\eta)= & \frac{1}{2 \pi} \int_{0}^{2 \pi} \log \left|f\left(R e^{i \phi}\right)\right| \frac{R e^{i \phi}+z+\eta}{R e^{i \phi}-z-\eta} d \phi-\sum_{\left|a_{\nu}\right|<R} \log \frac{R^{2}-\bar{a}_{\nu}(z+\eta)}{R\left(z+\eta-a_{\nu}\right)} \\
& +\sum_{\left|b_{\mu}\right|<R} \log \frac{R^{2}-\bar{b}_{\mu}(z+\eta)}{R\left(z+\eta-b_{\mu}\right)}+i C_{\eta}
\end{aligned}
$$


where

$$
C_{\eta}=\arg f(0)-\sum_{\left|b_{\mu}\right|<R} \arg \left(-\frac{R}{b_{\mu}}\right)+\sum_{\left|a_{\nu}\right|<R} \arg \left(-\frac{R}{a_{\nu}}\right)+2 m_{z, \eta} \pi .
$$

We subtract (3.10) from (3.12) and keep in mind that the integers $m_{z, \eta}, m_{z}$ in each step of the calculations below depend on the choice of the logarithms as mentioned in Remark 3.2. This yields

$$
\begin{aligned}
\log \frac{f(z+\eta)}{f(z)}= & \frac{1}{2 \pi} \int_{0}^{2 \pi} \log \left|f\left(R e^{i \phi}\right)\right| \frac{2 \eta R e^{i \phi}}{\left(R e^{i \phi}-z-\eta\right)\left(R e^{i \phi}-z\right)} d \phi \\
& +\sum_{\left|a_{\nu}\right|<R}\left[\log \frac{R^{2}-\bar{a}_{\nu} z}{R\left(z-a_{\nu}\right)}-\log \frac{R^{2}-\bar{a}_{\nu}(z+\eta)}{R\left(z+\eta-a_{\nu}\right)}\right] \\
& +\sum_{\left|b_{\mu}\right|<R}\left[\log \frac{R^{2}-\bar{b}_{\mu}(z+\eta)}{R\left(z+\eta-b_{\mu}\right)}-\log \frac{R^{2}-\bar{b}_{\mu} z}{R\left(z-b_{\mu}\right)}\right]+2 \pi\left(m_{z, \eta}-m_{z}\right) i \\
= & \frac{1}{2 \pi} \int_{0}^{2 \pi} \log \left|f\left(R e^{i \phi}\right)\right| \frac{2 \eta R e^{i \phi}}{\left(R e^{i \phi}-z-\eta\right)\left(R e^{i \phi}-z\right)} d \phi \\
& -\sum_{\left|a_{\nu}\right|<R} \log \left[\left(\frac{R^{2}-\bar{a}_{\nu}(z+\eta)}{R^{2}-\bar{a}_{\nu} z}\right)\left(\frac{z-a_{\nu}}{z+\eta-a_{\nu}}\right)\right] \\
& +\sum_{\left|b_{\mu}\right|<R} \log \left[\left(\frac{R^{2}-\bar{b}_{\mu}(z+\eta)}{R^{2}-\bar{b}_{\mu} z}\right)\left(\frac{z-b_{\mu}}{z+\eta-b_{\mu}}\right)\right]+2 \pi\left(m_{z, \eta}-m_{z}\right) i \\
= & \frac{1}{2 \pi} \int_{0}^{2 \pi} \log \left|f\left(R e^{i \phi}\right)\right| \frac{2 \eta R e^{i \phi}}{\left(R e^{i \phi}-z-\eta\right)\left(R e^{i \phi}-z\right)} d \phi \\
& -\sum_{\left|a_{\nu}\right|<R} \log \left(1-\frac{\bar{a}_{\nu} \eta}{R^{2}-\bar{a}_{\nu} z}\right)+\sum_{\left|a_{\nu}\right|<R} \log \left(1+\frac{\eta}{z-a_{\nu}}\right) \\
& +\sum_{\left|b_{\mu}\right|<R} \log \left(1-\frac{\bar{b}_{\mu} \eta}{R^{2}-\bar{b}_{\mu} z}\right)-\sum_{\left|b_{\mu}\right|<R} \log \left(1+\frac{\eta}{z-b_{\mu}}\right) \\
& +2 \pi\left(m_{z, \eta}-m_{z}\right) i .
\end{aligned}
$$

Differentiating the Poisson-Jensen formula (3.10) yields

$$
\begin{aligned}
\frac{f^{\prime}(z)}{f(z)}=\frac{1}{2 \pi} & \int_{0}^{2 \pi} \log \left|f\left(R e^{i \phi}\right)\right| \frac{2 R e^{i \phi}}{\left(R e^{i \phi}-z\right)^{2}} d \phi \\
& +\sum_{\left|a_{\nu}\right|<R} \frac{\bar{a}_{\nu}}{R^{2}-\bar{a}_{\nu} z}-\sum_{\left|b_{\mu}\right|<R} \frac{\bar{b}_{\mu}}{R^{2}-\bar{b}_{\mu} z} \\
& +\sum_{\left|a_{\nu}\right|<R} \frac{1}{z-a_{\nu}}-\sum_{\left|b_{\mu}\right|<R} \frac{1}{z-b_{\mu}} .
\end{aligned}
$$


We multiply the left hand side of (3.15) by $\eta$ and subtract the product from the left hand side of (3.14) with the assumption $|z|=r<R-|\eta|$. This gives

$$
\begin{aligned}
\log \frac{f(z+\eta)}{f(z)}-\eta \frac{f^{\prime}(z)}{f(z)}=\frac{\eta^{2}}{\pi} & \int_{0}^{2 \pi} \log \left|f\left(R e^{i \phi}\right)\right| \frac{R e^{i \phi}}{\left(R e^{i \phi}-z-\eta\right)\left(R e^{i \phi}-z\right)^{2}} d \phi \\
& -\sum_{\left|a_{\nu}\right|<R}\left[\log \left(1-\frac{\bar{a}_{\nu} \eta}{R^{2}-\bar{a}_{\nu} z}\right)+\frac{\bar{a}_{\nu} \eta}{R^{2}-\bar{a}_{\nu} z}\right] \\
& +\sum_{\left|b_{\mu}\right|<R}\left[\log \left(1-\frac{\bar{b}_{\mu} \eta}{R^{2}-\bar{b}_{\mu} z}\right)+\frac{\bar{b}_{\mu} \eta}{R^{2}-\bar{b}_{\mu} z}\right] \\
& +\sum_{\left|a_{\nu}\right|<R}\left[\log \left(1+\frac{\eta}{z-a_{\nu}}\right)-\frac{\eta}{z-a_{\nu}}\right] \\
& -\sum_{\left|b_{\mu}\right|<R}\left[\log \left(1+\frac{\eta}{z-b_{\mu}}\right)-\frac{\eta}{z-b_{\mu}}\right] \\
& +2 \pi\left(m_{z, \eta}-m_{z}\right) i .
\end{aligned}
$$

We distinguish two cases depending on the exponent of convergence of the zeros and poles of $f(z)$ in the rest of the proof. We first consider the case $\lambda=$ $\max \left\{\lambda^{\prime}, \lambda^{\prime \prime}\right\}<1$.

We may now choose the branches of the logarithms on the right hand side of (3.16) as in Lemma 3.2. Therefore we obtain

$$
\begin{aligned}
\mid 2 \pi\left(m_{z}-m_{z, \eta}\right) i+\log \frac{f(z+\eta)}{f(z)}- & \eta \frac{f^{\prime}(z)}{f(z)}\left|\leq \frac{R|\eta|^{2}}{\pi(R-r-|\eta|)^{3}} \int_{0}^{2 \pi}\right| \log \left|f\left(R e^{i \phi}\right)\right| \mid d \phi \\
& +O\left[\sum_{\left|a_{\nu}\right|<R}\left(\left|\frac{\bar{a}_{\nu} \eta}{R^{2}-\bar{a}_{\nu} z}\right|^{2}+\left|\frac{\bar{a}_{\nu} \eta}{R^{2}-\bar{a}_{\nu} z-\bar{a}_{\nu} \eta}\right|^{2}\right)\right. \\
& +\sum_{\left|b_{\mu}\right|<R}\left(\left|\frac{\bar{b}_{\mu} \eta}{R^{2}-\bar{b}_{\mu} z}\right|^{2}+\left|\frac{\bar{b}_{\mu} \eta}{R^{2}-\bar{b}_{\mu} z-\bar{b}_{\mu} \eta}\right|^{2}\right) \\
& +\sum_{\left|a_{\nu}\right|<R}\left(\left|\frac{\eta}{z-a_{\nu}}\right|^{2}+\left|\frac{\eta}{z-a_{\nu}+\eta}\right|^{2}\right) \\
& \left.+\sum_{\left|b_{\mu}\right|<R}\left(\left|\frac{\eta}{z-b_{\mu}}\right|^{2}+\left|\frac{\eta}{\mid z-b_{\mu}+\eta}\right|^{2}\right)\right] .
\end{aligned}
$$

We first note that

$$
\int_{0}^{2 \pi}|\log | f\left(R e^{i \phi}\right)|| d \phi=2 \pi(m(R, f)+m(R, 1 / f)) .
$$


We next estimate the remaining terms in (3.17). We have

$$
\begin{aligned}
\sum_{\left|a_{\nu}\right|<R}\left(\left|\frac{\bar{a}_{\nu} \eta}{R^{2}-\bar{a}_{\nu} z}\right|^{2}+\right. & \left.\left|\frac{\bar{a}_{\nu} \eta}{R^{2}-\bar{a}_{\nu} z-\bar{a}_{\nu} \eta}\right|^{2}\right) \\
& \leq \sum_{\left|a_{\nu}\right|<R} \frac{2|\eta|^{2} R^{2}}{\left(R^{2}-R r-R|\eta|\right)^{2}}=\frac{2|\eta|^{2}}{(R-r-|\eta|)^{2}} n(R, 1 / f)
\end{aligned}
$$

and

$$
\begin{aligned}
\sum_{\left|b_{\mu}\right|<R}\left(\left|\frac{\bar{b}_{\mu} \eta}{R^{2}-\bar{b}_{\mu} z}\right|^{2}\right. & \left.+\left|\frac{\bar{b}_{\mu} \eta}{R^{2}-\bar{b}_{\mu} z-\bar{b}_{\mu} \eta}\right|^{2}\right) \\
& \leq \sum_{\left|b_{\mu}\right|<R} \frac{2|\eta|^{2} R^{2}}{\left(R^{2}-R r-R|\eta|\right)^{2}}=\frac{2|\eta|^{2}}{(R-r-|\eta|)^{2}} n(R, f)
\end{aligned}
$$

On the other hand, it is elementary that when $R^{\prime}>R>1$, we have

$$
\begin{aligned}
N\left(R^{\prime}, f\right) & \geq \int_{R}^{R^{\prime}} \frac{n(t, f)-n(0, f)}{t} d t+n(0, f) \log R^{\prime} \\
& \geq n(R, f) \int_{R}^{R^{\prime}} \frac{d t}{t}-n(0, f) \int_{R}^{R^{\prime}} \frac{d t}{t}+n(0, f) \log R^{\prime} \\
& \geq n(R, f) \frac{R^{\prime}-R}{R^{\prime}} .
\end{aligned}
$$

Then for $R>1$, we have

$$
n(R, f) \leq 2 N(2 R, f)
$$

and similarly

$$
n(R, 1 / f) \leq 2 N(2 R, 1 / f) .
$$

We may now combine (3.17)-(3.20), (3.22) and (3.23) to get

$$
\begin{aligned}
\mid 2 \pi\left(m_{z}\right. & \left.-m_{z, \eta}\right) i+\log \frac{f(z+\eta)}{f(z)}-\eta \frac{f^{\prime}(z)}{f(z)} \mid \\
= & O\left[\frac{R}{(R-r-|\eta|)^{3}}(m(R, f)+m(R, 1 / f))\right. \\
& +\frac{1}{(R-r-|\eta|)^{2}}(N(2 R, f)+N(2 R, 1 / f)) \\
& \left.+\sum_{\left|c_{k}\right|<R}\left(\frac{1}{\left|z-c_{k}\right|^{2}}+\frac{1}{\left|z-c_{k}+\eta\right|^{2}}\right)\right],
\end{aligned}
$$


where $\left(c_{k}\right)_{k \in N}=\left(a_{\nu}\right)_{\nu \in N} \cup\left(b_{\mu}\right)_{\mu \in N}$. Let $r>\max \left\{|\eta|, \frac{1}{3}\right\}$ and $R=3 r$. Then it follows from (3.24) and the finite order $\sigma$ assumption on $f$ that

$$
\begin{aligned}
2 \pi\left(m_{z}-m_{z, \eta}\right) i & +\log \frac{f(z+\eta)}{f(z)}-\eta \frac{f^{\prime}(z)}{f(z)} \\
& =O\left(r^{\sigma-2+\varepsilon}\right)+O\left(\sum_{\left|c_{k}\right|<3 r} \frac{1}{\left|z-c_{k}\right|^{2}}+\frac{1}{\left|z-c_{k}+\eta\right|^{2}}\right) \\
& =O\left(r^{\sigma-2+\varepsilon}\right)+O\left(\sum_{\left|d_{k}\right|<4 r} \frac{1}{\left|z-d_{k}\right|^{2}}\right),
\end{aligned}
$$

where $\left(d_{k}\right)_{k \in N}=\left(c_{k}\right)_{k \in N} \cup\left(c_{k}-\eta\right)_{k \in N}$.

We now estimate the second summand in (3.25) via the Cartan lemma in the spirit in [12. Let $n(t)$ denote the number of the points $d_{k}$ that lie in $|z|<t$. Then

$$
n(t) \leq n(t+|\eta|, f)+n(t+|\eta|, 1 / f) .
$$

We suppose that $h$ is any fixed positive integer and that $z$ is confined to the annulus

$$
4^{h} \leq|z|=r \leq 4^{h+1} .
$$

Set $p=n\left(4^{h+2}\right), B=\frac{4^{h}}{h^{2}}$, and apply Lemma 3.4 to the points $d_{1}, d_{2}, \cdots, d_{p}$, to conclude that there exists a finite collection of closed disks $D_{1}, D_{2}, \cdots, D_{q}$, whose radii has a total sum equal to $2 B$, such that if $z \notin D_{j}$ for $j=1,2, \cdots, q$, then there is a permutation of the points $d_{1}, d_{2}, \cdots, d_{p}$, say, $\hat{d}_{1}, \hat{d}_{2}, \cdots, \hat{d}_{p}$, that satisfies

$$
\left|z-\hat{d}_{k}\right|>B \frac{k}{p}, \quad k=1,2, \cdots, p .
$$

We note here that $q$ and $D_{1}, D_{2}, \cdots, D_{q}$ depend on $p$ and then depend on $h$. Hence if $z \notin D_{j}$ for $j=1,2, \cdots, q$, we have from (3.27) and (3.28) that

$$
\begin{aligned}
\sum_{\left|d_{k}\right|<4 r} \frac{1}{\left|z-d_{k}\right|^{2}} & \leq \sum_{k=1}^{p} \frac{1}{\left|z-d_{k}\right|^{2}}=\sum_{k=1}^{p} \frac{1}{\left|z-\hat{d}_{k}\right|^{2}} \\
& \leq \sum_{k=1}^{p} \frac{p^{2}}{B^{2} k^{2}} \leq \frac{p^{2}}{B^{2}} \sum_{k=1}^{\infty} \frac{1}{k^{2}} \\
& \leq \frac{16[n(16 r)]^{2} \log ^{4} r}{r^{2} \log ^{4} 4} \sum_{k=1}^{\infty} \frac{1}{k^{2}} .
\end{aligned}
$$

We deduce from (3.26) and (3.29) that

$$
\sum_{\left|d_{k}\right|<4 r} \frac{1}{\left|z-d_{k}\right|^{2}}=O\left(r^{2 \lambda-2+\varepsilon}\right)+O\left(r^{2 \lambda^{\prime}-2+\varepsilon}\right) .
$$

It remains to consider the exceptional sets arising from the discs in the Cartan lemma. For each $h$, we define (it has been mentioned that $q$ and $D_{1}, D_{2}, \cdots, D_{q}$ depend on $h$ )

$$
Y_{h}=\left\{r: z \in \bigcup_{j=1}^{q} D_{j} \text { such that }|z|=r\right\}
$$

and

$$
E_{h}=Y_{h} \cap\left[4^{h}, 4^{h+1}\right] .
$$


Then

$$
\int_{E_{h}} 1 d x \leq \int_{Y_{h}} 1 d x \leq 4 B=\frac{4^{h+1}}{h^{2}} .
$$

Set

$$
E=\left\{\bigcup_{h=0}^{\infty} E_{h}\right\} \cap(1, \infty)
$$

We deduce, by combining (3.25) and (3.30), that

$$
2 \pi\left(m_{z}-m_{z, \eta}\right) i+\log \frac{f(z+\eta)}{f(z)}=\eta \frac{f^{\prime}(z)}{f(z)}+O\left(r^{\beta+\varepsilon}\right)
$$

holds for all $z$ satisfying $|z|=r \notin[0,1] \cup E$. The sizes of the exceptional sets can be calculated from (3.32)-(3.33). We have

$$
\int_{E} \frac{1}{t} d t \leq \sum_{h=1}^{\infty} \int_{E_{h}} \frac{1}{t} d t \leq 4 \sum_{h=1}^{\infty} \frac{1}{h^{2}}<\infty ;
$$

that is, $E$ has finite logarithmic measure. This completes the proof when $\lambda<1$.

We now consider the remaining case when $\lambda=\max \left\{\lambda^{\prime}, \lambda^{\prime \prime}\right\} \geq 1$. We shall appeal directly to (3.16) with $r=|z|<R-|\eta|$. This together with (3.4) and (3.18) yields, as in the case above when $\lambda<1$,

$$
\begin{aligned}
\mid 2 \pi\left(m_{z}-m_{z, \eta}\right) i+\log \frac{f(z+\eta)}{f(z)}- & \eta \frac{f^{\prime}(z)}{f(z)} \mid \leq O\left[\frac{R(m(R, f)+m(R, 1 / f))}{(R-r-|\eta|)^{3}}\right] \\
& +\sum_{\left|c_{\mu}\right|<R}\left[\left|\log \left(1-\frac{\bar{c}_{\mu} \eta}{R^{2}-\bar{c}_{\mu} z}\right)\right|+\left|\frac{\bar{c}_{\mu} \eta}{R^{2}-\bar{c}_{\mu} z}\right|\right] \\
& +\sum_{\left|c_{\mu}\right|<R}\left[\left|\log \left(1+\frac{\eta}{z-c_{\mu}}\right)\right|+\left|\frac{\eta}{z-c_{\mu}}\right|\right] \\
\leq & O\left[\frac{R}{(R-r-|\eta|)^{3}}(m(R, f)+m(R, 1 / f))\right] \\
& +O\left[\sum_{\left|c_{\mu}\right|<R}\left(\left|\frac{\bar{c}_{\mu} \eta}{R^{2}-\bar{c}_{\mu} z}\right|+\left|\frac{\bar{c}_{\mu} \eta}{R^{2}-\bar{c}_{\mu} z-\bar{c}_{\mu} \eta}\right|\right)\right] \\
& +O\left[\sum_{\left|c_{\mu}\right|<R}\left(\left|\frac{\eta}{z-c_{\mu}}\right|+\left|\frac{\eta}{z+\eta-c_{\mu}}\right|\right)\right] .
\end{aligned}
$$

We then choose $R=3 r, r>\max \{|\eta|, 1 / 3\}$. We also choose $|z|$ as in (3.27), $p=n\left(4^{h+2}\right)$. Hence

$$
\begin{aligned}
\sum_{\left|c_{\mu}\right|<R}\left(\left|\frac{\bar{c}_{\mu} \eta}{R^{2}-\bar{c}_{\mu} z}\right|\right. & \left.+\left|\frac{\bar{c}_{\mu} \eta}{R^{2}-\bar{c}_{\mu} z-\bar{c}_{\mu} \eta}\right|\right) \leq \sum_{\left|d_{\mu}\right|<4 r}\left|\frac{\bar{d}_{\mu} \eta}{(4 r)^{2}-\bar{d}_{\mu} z}\right| \\
& \leq \sum_{\nu=1}^{p} \frac{|\eta|}{4 r-r}=p \frac{|\eta|}{3 r}=O\left(r^{\lambda-1+\varepsilon}\right) .
\end{aligned}
$$


We apply the Cartan argument similar to (3.27)-(3.29) as in the previous case when $\lambda<1$ to obtain

$$
\begin{aligned}
\sum_{\left|c_{\mu}\right|<R}\left(\left|\frac{\eta}{z-c_{\mu}}\right|\right. & \left.+\left|\frac{\eta}{z+\eta-c_{\mu}}\right|\right) \leq \sum_{\left|d_{\mu}\right|<4 r}\left|\frac{\eta}{z-d_{\mu}}\right| \\
& \leq|\eta| \sum_{\nu=1}^{p} \frac{1}{\left|z-\hat{d}_{k}\right|} \leq|\eta| \sum_{\nu=1}^{p} \frac{p}{B} \frac{1}{\nu} \\
& \leq|\eta| p \frac{h^{2}}{4^{h}} \sum_{\nu=1}^{p} \frac{1}{\nu} \leq|\eta| \frac{4 n(16 r)}{r}\left(\frac{\log r}{\log 4}\right)^{2} \log n(16 r)=O\left(r^{\lambda-1+\varepsilon}\right)
\end{aligned}
$$

Combining (3.36), (3.37) and (3.38) yields

$$
\begin{aligned}
\left|2 \pi\left(m_{z}-m_{z, \eta}\right) i+\log \frac{f(z+\eta)}{f(z)}-\eta \frac{f^{\prime}(z)}{f(z)}\right| \leq & O\left(r^{\sigma-2+\varepsilon}\right)+O\left(\sum_{\left|d_{\mu}\right|<4 r}\left|\frac{\bar{d}_{\mu} \eta}{R^{2}-\bar{d}_{\mu} z}\right|\right) \\
& +O\left(\sum_{\left|d_{\mu}\right|<4 r}\left|\frac{\eta}{z-d_{\mu}}\right|\right) \\
\leq & O\left(r^{\sigma-2+\varepsilon}\right)+O\left(r^{\lambda-1+\varepsilon}\right),
\end{aligned}
$$

which holds outside a set of finite logarithmic measure. This completes the proof for the case when $\lambda \geq 1$ and hence that of the theorem.

\section{First ORDER DIFFERENCE QUOTIENTS ESTIMATES}

\subsection{Main results.}

Theorem 4.1. Let $f$ be a meromorphic function of order $\sigma(f)=\sigma<1$, and let $\eta$ be a fixed, non-zero number. Then for any $\varepsilon>0$, there exists a set $E \subset(1, \infty)$ that depends on $f$ and has finite logarithmic measure, such that for all $z$ satisfying $|z|=r \notin E \cup[0,1]$, we have

$$
\frac{\Delta f(z)}{f(z)}=\frac{f(z+\eta)-f(z)}{f(z)}=\eta \frac{f^{\prime}(z)}{f(z)}+O\left(r^{2 \sigma-2+\varepsilon}\right) .
$$

Remark 4.1. We note that when $\eta=1$, then (4.1) assumes the form

$$
\frac{\Delta f(z)}{f(z)}=\frac{f(z+1)-f(z)}{f(z)}=\frac{f^{\prime}(z)}{f(z)}+O\left(r^{2 \sigma-2+\varepsilon}\right) .
$$

This estimate will be extended to higher order difference quotients in the next section.

We next consider an example $f(z)$ showing that when $\sigma(f)=1 / 2$, then the exponent " $2 \sigma-2=-1$ " that appears in the error term of (4.1) is best possible.

Example 4.2. Let

$$
f(z)=\cos \sqrt{z},
$$

which is clearly an entire function of order $1 / 2$. Then there is a set $F$ of positive real numbers of infinite logarithmic measure, such that for all $x \in F$, we have

$$
\left|\frac{\Delta f(x)}{f(x)}-\frac{f^{\prime}(x)}{f(x)}\right| \geq \frac{1}{16 x}(1+o(1)) .
$$


We first compute the numerators on the left hand side of (4.4) by using trigonometric identities. This gives

$$
\begin{aligned}
& \Delta f(z)-f^{\prime}(z)=\cos \sqrt{z+1}-\cos \sqrt{z}+\frac{\sin \sqrt{z}}{2 \sqrt{z}} \\
& =-2 \sin \left(\frac{\sqrt{z+1}+\sqrt{z}}{2}\right) \sin \left(\frac{\sqrt{z+1}-\sqrt{z}}{2}\right)+\frac{\sin \sqrt{z}}{2 \sqrt{z}} \\
& =\frac{\sin \sqrt{z}}{2 \sqrt{z}}-\frac{1}{2 \sqrt{z}} \sin \left(\frac{\sqrt{z+1}+\sqrt{z}}{2}\right)+\frac{1}{2 \sqrt{z}} \sin \left(\frac{\sqrt{z+1}+\sqrt{z}}{2}\right) \\
& -2 \sin \left(\frac{\sqrt{z+1}+\sqrt{z}}{2}\right) \sin \left(\frac{\sqrt{z+1}-\sqrt{z}}{2}\right) \\
& =\frac{1}{2 \sqrt{z}} \cdot 2 \cos \left(\frac{\sqrt{z}+\frac{\sqrt{z+1}+\sqrt{z}}{2}}{2}\right) \sin \left(\frac{\sqrt{z}-\frac{\sqrt{z+1}+\sqrt{z}}{2}}{2}\right) \\
& +\sin \left(\frac{\sqrt{z+1}+\sqrt{z}}{2}\right)\left[\frac{1}{2 \sqrt{z}}-2 \sin \left(\frac{\sqrt{z+1}-\sqrt{z}}{2}\right)\right] \\
& =-\frac{1}{\sqrt{z}} \cos \left(\frac{\sqrt{z+1}+3 \sqrt{z}}{4}\right) \sin \left(\frac{1}{4(\sqrt{z+1}+\sqrt{z})}\right) \\
& +\sin \left(\frac{\sqrt{z+1}+\sqrt{z}}{2}\right)\left[\frac{1}{2 \sqrt{z}}-2 \sin \left(\frac{1}{2(\sqrt{z+1}+\sqrt{z})}\right)\right] \text {. }
\end{aligned}
$$

We now consider $z=x>0$ and let $x \rightarrow+\infty$. This yields

$$
\begin{aligned}
\Delta f(x)-f^{\prime}(x)= & -\frac{1}{\sqrt{x}} \cos \left(\frac{\sqrt{x+1}+3 \sqrt{x}}{4}\right) \cdot \frac{(1+o(1))}{8 \sqrt{x}} \\
& +\sin \left(\frac{\sqrt{x+1}+\sqrt{x}}{2}\right)\left[\frac{1}{2 \sqrt{x}}-\frac{1}{(\sqrt{x+1}+\sqrt{x})}\right. \\
& \left.+O\left(\frac{1}{(\sqrt{x+1}+\sqrt{x})}\right)^{3}\right] \\
= & -\frac{1}{8 x} \cos \left(\frac{\sqrt{x+1}+3 \sqrt{x}}{4}\right)(1+o(1)) \\
& +\sin \left(\frac{\sqrt{x+1}+\sqrt{x}}{2}\right)\left[\frac{1}{2 \sqrt{x}(\sqrt{x+1}+\sqrt{x})^{2}}+O\left(x^{-3 / 2}\right)\right] \\
= & -\frac{1}{8 x}(1+o(1)) \cos \left(\frac{\sqrt{x+1}+3 \sqrt{x}}{4}\right)+O\left(x^{-3 / 2}\right) .
\end{aligned}
$$

We now let the subset $F$ of $\mathbf{R}$ be in the form

$$
F=\bigcup_{n=1}^{\infty}\left[(2 \pi n-\pi / 3)^{2},(2 \pi n+\pi / 3)^{2}\right],
$$

which clearly has infinite logarithmic measure. We notice that for $x \in F$ the function $\cos \sqrt{x}$ satisfies the inequality

$$
\frac{1}{2} \leq \cos \sqrt{x} \leq 1 .
$$


Combining (4.6) and (4.8) yields, when $x \in F$ and $x \rightarrow \infty$,

$$
\frac{\Delta f(x)}{f(x)}-\frac{f^{\prime}(x)}{f(x)} \leq-\frac{1}{8 x}(1+o(1)) \cos \left(\frac{\sqrt{x+1}+3 \sqrt{x}}{4}\right)+O\left(x^{-3 / 2}\right) .
$$

It remains to consider the leading term on the right hand side of (4.9). We further notice that

$$
\begin{aligned}
\cos \left(\frac{\sqrt{x+1}+3 \sqrt{x}}{4}\right)-\cos \sqrt{x} & =-2 \sin \left(\frac{\frac{\sqrt{x+1}+3 \sqrt{x}}{4}+\sqrt{x}}{2}\right) \sin \left(\frac{\frac{\sqrt{x+1}+3 \sqrt{x}}{4}-\sqrt{x}}{2}\right) \\
& =O(1) \cdot \sin \left(\frac{1}{8(\sqrt{x+1}+\sqrt{x})}\right) \\
& =o(1) .
\end{aligned}
$$

Thus we deduce from (4.10), for all $x \in F$, that

$$
\cos \left(\frac{\sqrt{x+1}+3 \sqrt{x}}{4}\right)=\cos \sqrt{x}+o(1) \geq \frac{1}{2}+o(1) .
$$

We can easily see that (4.4) follows from (4.11) and (4.9).

4.2. Proof of Theorem 4.1. We require the classical estimate of Gundersen mentioned in the Introduction.

Lemma 4.1 ([12]). Let $f$ be a meromorphic function of finite order $\sigma(f)=\sigma$. Then for any $\varepsilon>0$, there exists a set $E \subset(1, \infty)$ that depends on $f$ and has finite logarithmic measure, such that for all $z$ satisfying $|z|=r \notin E \cup[0,1]$, we have

$$
\left|\frac{f^{\prime}(z)}{f(z)}\right| \leq|z|^{\sigma-1+\varepsilon}
$$

Proof. Given an arbitrary $\varepsilon$ such that $0<\varepsilon<1-\sigma$, Theorem 2.1 and Lemma 4.1 imply that there exists a set $F \subset(1, \infty)$ that depends only on $f$ and has finite logarithmic measure, such that for all $z$ satisfying $|z|=r \notin F \cup[0,1]$,

$$
2 n_{z} \pi i+\log \frac{f(z+\eta)}{f(z)}=O\left(r^{\sigma-1+\varepsilon}\right)=o(1)
$$

Then there exists a constant $A>0$, such that for all $z$ satisfying $|z|=r \notin F \cup[0, A]$,

$$
\left|2 n_{z} \pi i+\log \frac{f(z+\eta)}{f(z)}\right| \leq 1
$$

Since

$$
e^{w}-1=w+O\left(w^{2}\right), \quad|w| \leq 1,
$$


we therefore deduce from (4.13), (4.14), 4.15) and (2.2) that for all $z$ satisfying $|z|=r \notin F \cup[0, A]$,

$$
\begin{aligned}
\frac{f(z+\eta)-f(z)}{f(z)} & =e^{2 n_{z} \pi i+\log \frac{f(z+\eta)}{f(z)}}-1 \\
& =2 n_{z} \pi i+\log \frac{f(z+\eta)}{f(z)}+O\left(2 n_{z} \pi i+\log \frac{f(z+\eta)}{f(z)}\right)^{2} \\
& =\eta \frac{f^{\prime}(z)}{f(z)}+O\left(r^{2 \sigma-2+\varepsilon}\right)+O\left(\left(r^{\sigma-1+\varepsilon}\right)^{2}\right) \\
& =\eta \frac{f^{\prime}(z)}{f(z)}+O\left(r^{2 \sigma-2+2 \varepsilon}\right) .
\end{aligned}
$$

Now let $E=F \cup[1, A]$. Then $E$ has finite logarithmic measure and hence (4.1) holds for all $z$ satisfying $|z|=r \notin E \cup[0,1]$.

\section{Higher ORDER DIFFERENCE QUOTIENTS ESTIMATES}

\subsection{Main results.}

Theorem 5.1. Let $f$ be a meromorphic function of order $\sigma(f)=\sigma<1$. Then for each given $\varepsilon>0$, and integers $0 \leq j<k$, there exists a set $E \subset(1, \infty)$ that depends on $f$, and it has finite logarithmic measure, such that for all $z$ satisfying $|z|=r \notin E \cup[0,1]$, we have

$$
\left|\frac{\Delta^{k} f(z)}{\Delta^{j} f(z)}\right| \leq|z|^{(k-j)(\sigma-1)+\varepsilon}
$$

We deduce the following consequence from (5.1). This is a higher order extension of Theorem 4.1 .

Theorem 5.2. Let $f$ be a meromorphic function of order $\sigma(f)=\sigma<1$, and let $\eta$ be a fixed, non-zero number. Then for any $\varepsilon>0, k \in N$, there exists a set $E \subset(1, \infty)$ that depends on $f$ and has finite logarithmic measure, such that for all $z$ satisfying $|z|=r \notin E \cup[0,1]$, we have

$$
\frac{\Delta^{k} f(z)}{f(z)}=\eta^{k} \frac{f^{(k)}(z)}{f(z)}+O\left(r^{(k+1)(\sigma-1)+\varepsilon}\right) .
$$

We need the following elementary lemma to prove the theorems.

Lemma 5.1. Let $f$ be a finite order meromorphic function. Then for each $k \in N$,

$$
\sigma\left(\Delta^{k} f\right) \leq \sigma(f)
$$

Proof. Let $\sigma=\sigma(f)$. It is sufficient to prove the case when $k=1$. We recall that the authors prove

$$
T(r, f(z+\eta)) \sim T(r, f(z))+O\left(r^{\sigma-1+\varepsilon}\right)+O(\log r)
$$

in ([8, Thm. 1]). Thus, we see immediately from (5.4) that $\sigma(f(z+\eta))=\sigma(f(z))$ holds. Hence

$$
\sigma(\Delta f(z))=\sigma(f(z+1)-f(z)) \leq \max \{\sigma(f(z+1)), \sigma(f(z))\}=\sigma(f(z)) .
$$

Repeating the above argument $k-1$ times would yield (5.3).

Remark 5.1. It is easy to see that the inequality $\sigma(f) \leq \sigma\left(\Delta^{k} f\right)$ does not hold for general meromorphic functions. For example, one can take $g(z)=\Gamma^{\prime}(z) / \Gamma(z)$. 


\subsection{Proof of Theorem 5.1.}

Proof. Let $j$ be a non-negative integer amongst the set $\{0,1, \cdots, k-1\}$. We deduce from (4.1), (4.12) and (5.3) that for each such $j$, there exists a set $E_{j} \subset(1, \infty)$ that depends on $f$ and has finite logarithmic measure, such that for all $z$ satisfying $|z|=r \notin E_{j} \cup[0,1]$,

$$
\left|\frac{\Delta^{j+1} f(z)}{\Delta^{j} f(z)}\right| \leq|z|^{\sigma_{j}-1+\varepsilon} \leq|z|^{\sigma-1+\varepsilon}
$$

where $\sigma_{j}=\sigma\left(\Delta^{j} f\right)$. Now let $E=\bigcup_{j=0}^{k-1} E_{j}$, which clearly has finite logarithmic measure. Hence, we have, after applying (5.6) repeatedly, that

$$
\left|\frac{\Delta^{k} f(z)}{f(z)}\right|=\left|\frac{\Delta^{k} f(z)}{\Delta^{k-1} f(z)} \cdot \frac{\Delta^{k-1} f(z)}{\Delta^{k-2} f(z)} \cdots \frac{\Delta f(z)}{f(z)}\right| \leq\left(|z|^{\sigma-1+\varepsilon}\right)^{k} \leq|z|^{k \sigma-k+k \varepsilon}
$$

holds for all $z$ satisfying $|z|=r \notin E \cup[0,1]$. Since $\varepsilon$ is arbitrary, this completes the proof of (5.1) when $j=0$. Let $G=\Delta^{j} f(j<k)$. Then Lemma 5.1 implies that $\sigma(G) \leq \sigma(f)$. Thus (5.7) asserts that there is an exceptional set $E$ of finite logarithmic measure such that for all $z$ satisfying $|z|=r \notin E$, we have

$$
\left|\frac{\Delta^{k} f(z)}{\Delta^{j} f(z)}\right|=\left|\frac{\Delta^{k-j} G(z)}{G(z)}\right| \leq|z|^{(k-j)(\sigma-1)+\varepsilon} .
$$

This completes the proof of the theorem.

\subsection{Proof of Theorem 5.2 ,}

Proof. The case when $k=1$ is just equation (4.1) in Theorem 4.1. We shall prove (5.2) by induction on $k$. We assume that (5.2) is true for all $k, k \leq j$. We deduce from Theorem 4.1, Lemma 4.1, Theorem 5.1, Lemma 5.1, and the fact that $\sigma\left(f^{\prime}\right)=\sigma(f)$, that there exists a set $E \subset(1, \infty)$ that depends on $f$, which has finite logarithmic measure, such that for all $z$ satisfying $|z|=r \notin E \cup[0,1]$,

$$
\begin{aligned}
& \frac{\Delta^{j+1} f(z)}{f(z)}=\frac{\Delta^{j+1} f(z)}{\Delta^{j} f(z)} \cdot \frac{\Delta^{j} f(z)}{f(z)}=\left[\frac{\left(\Delta^{j} f\right)^{\prime}(z)}{\Delta^{j} f(z)}+O\left(r^{2 \sigma(f)-2+\varepsilon}\right)\right] \cdot \frac{\Delta^{j} f(z)}{f(z)} \\
& =\frac{\Delta^{j}\left(f^{\prime}(z)\right)}{\Delta^{j} f(z)} \cdot \frac{\Delta^{j} f(z)}{f(z)}+O\left(r^{2 \sigma(f)-2+\varepsilon}\right) \cdot \frac{\Delta^{j} f(z)}{f(z)} \\
& =\frac{\Delta^{j}\left(f^{\prime}(z)\right)}{f(z)}+O\left(r^{(j+2)(\sigma(f)-1)+2 \varepsilon}\right) \\
& =\frac{\Delta^{j}\left(f^{\prime}(z)\right)}{f^{\prime}(z)} \cdot \frac{f^{\prime}(z)}{f(z)}+O\left(r^{(j+2)(\sigma(f)-1)+2 \varepsilon}\right) \\
& =\left[\frac{\left(f^{\prime}\right)^{(j)}(z)}{f^{\prime}(z)}+O\left(r^{(j+1)\left(\sigma\left(f^{\prime}\right)-1\right)+\varepsilon}\right)\right] \cdot \frac{f^{\prime}(z)}{f(z)}+O\left(r^{(j+2)(\sigma(f)-1)+2 \varepsilon}\right) \\
& =\frac{f^{(j+1)}(z)}{f^{\prime}(z)} \cdot \frac{f^{\prime}(z)}{f(z)}+O\left(r^{(j+1)(\sigma(f)-1)+\varepsilon}\right) \cdot \frac{f^{\prime}(z)}{f(z)}+O\left(r^{(j+2)(\sigma(f)-1)+2 \varepsilon}\right) \\
& =\frac{f^{(j+1)}(z)}{f(z)}+O\left(r^{(j+2)(\sigma(f)-1)+2 \varepsilon}\right) .
\end{aligned}
$$

That is, the case $k=j+1$ is true, and the proof is complete. 


\section{Difference Wiman-Valiron type estimates}

Let $f(z)=\sum_{n=0}^{\infty} a_{n} z^{n}$ be an entire function in the complex plane. If $r>0$, we define the maximum modulus $M(r, f)$ and maximal term $\mu(r, f)$ of $f$ by

$$
M(r, f)=\max _{|z|=r}|f(z)| \quad \text { and } \quad \mu(r, f)=\max _{n \geq 0}\left|a_{n}\right| r^{n},
$$

respectively. The central index $\nu(r, f)$ is the greatest exponent $m$ such that

$$
\left|a_{m}\right| r^{m}=\mu(r, f) .
$$

We note that $\nu(r, f)$ is a real, non-decreasing function of $r$.

We have the following fundamental result (see 27] and [32]) that relates the finite order of $f$ and its central index.

Lemma 6.1. If $f \not \equiv$ const. is an entire function of order $\sigma$, then

$$
\limsup _{r \rightarrow \infty} \frac{\log \nu(r, f)}{\log r}=\sigma .
$$

We next quote the classical result of Wiman-Valiron (see also [20]) in the form

Lemma 6.2 (He and Xiao [22, pp. 28-30]). Let $f$ be a transcendental entire function. Let $0<\varepsilon<\frac{1}{8}$ and $z$ be such that $|z|=r$ and that

$$
|f(z)|>M(r, f)(\nu(r, f))^{-\frac{1}{8}+\varepsilon}
$$

holds. Then there exists a set $E \subset(1, \infty)$ of finite logarithmic measure, such that

$$
\begin{aligned}
& \frac{f^{(k)}(z)}{f(z)}=\left(\frac{\nu(r, f)}{z}\right)^{k}\left(1+R_{k}(z)\right), \\
& R_{k}(z)=O\left((\nu(r, f))^{-\frac{1}{8}+\varepsilon}\right)
\end{aligned}
$$

holds for all $k \in \mathbf{N}$ and all $r \notin E \cup[0,1]$.

We deduce from Theorem 5.2 (6.3) and (6.4) in Lemma 6.2, the following.

Theorem 6.1. Let $f$ be a transcendental entire function of order $\sigma(f)=\sigma<1$, let $0<\varepsilon<\frac{1}{8}$ and $z$ be such that $|z|=r$, where

$$
|f(z)|>M(r, f)(\nu(r, f))^{-\frac{1}{8}+\varepsilon}
$$

holds. Then for each positive integer $k$, there exists a set $E \subset(1, \infty)$ that has finite logarithmic measure, such that for all $r \notin E \cup[0,1]$,

$$
\begin{aligned}
& \frac{\Delta^{k} f(z)}{f(z)}=\eta^{k}\left(\frac{\nu(r, f)}{z}\right)^{k}\left(1+O\left((\nu(r, f))^{-\frac{1}{8}+\varepsilon}\right)\right), \quad \text { if } \quad \sigma=0, \\
& \frac{\Delta^{k} f(z)}{f(z)}=\eta^{k}\left(\frac{\nu(r, f)}{z}\right)^{k}+O\left(r^{k \sigma-k-\gamma+\varepsilon}\right), \quad \text { if } \quad 0<\sigma<1,
\end{aligned}
$$

where $\gamma=\min \left\{\frac{1}{8} \sigma, 1-\sigma\right\}$. 


\section{ApPlichtions to Difference EQUations}

Theorem 7.1. Let $P_{0}(z), \cdots, P_{n}(z)$ be polynomials such that

$$
\max _{1 \leq j \leq n}\left\{\operatorname{deg} P_{j}\right\} \leq \operatorname{deg}\left(P_{0}\right) .
$$

Let $f(z)$ be a meromorphic solution to the difference equation

$$
P_{n}(z) \Delta^{n} f(z)+\cdots+P_{1}(z) \Delta f(z)+P_{0}(z) f(z)=0 .
$$

Then $\sigma(f) \geq 1$.

Proof. Let us first assume that a meromorphic solution $f$ has order $\sigma(f)<1$. We now write equation (7.2) in the form

$$
-1=\frac{P_{n}(z)}{P_{0}(z)} \frac{\Delta^{n} f(z)}{f(z)}+\cdots+\frac{P_{1}(z)}{P_{0}(z)} \frac{\Delta f(z)}{f(z)},
$$

and choose $\varepsilon$ such that $0<\varepsilon<1-\sigma(f)$. Theorem 5.1 asserts that there exists a set $E \subset(1,+\infty)$ of finite logarithmic measure, such that for all $|z|=r \notin E \cup[0,1]$,

$$
\frac{\Delta^{j} f(z)}{f(z)}=o(1)
$$

for $1 \leq j \leq n$. But

$$
\frac{P_{j}(z)}{P_{0}(z)}=O(1)
$$

as $|z| \rightarrow \infty$, for $1 \leq j \leq n$. Therefore, if we choose $|z|=r \notin E \cup[0,1]$ and $|z| \rightarrow \infty$, then it follows from (7.4) and (7.5) that we have a contradiction in (7.3).

We note that this generalizes our earlier result [8, Thm. 9.4], which we state as

Corollary 7.2. Let $Q_{0}(z), \cdots Q_{n}(z)$ be polynomials such that there exists an integer $\ell, 0 \leq \ell \leq n$ so that

$$
\operatorname{deg}\left(Q_{\ell}\right)>\max _{\substack{0 \leq \ell \leq n \\ j \neq \ell}}\left\{\operatorname{deg}\left(Q_{j}\right)\right\}
$$

holds. Suppose $f(z)$ is a meromorphic solution to

$$
Q_{n}(z) y(z+n)+\cdots+Q_{1}(z) y(z+1)+Q_{0} y(z)=0 .
$$

Then we have $\sigma(f) \geq 1$.

This is because if there is a polynomial coefficient of highest degree amongst the $Q_{0}(z), \cdots Q_{n}(z)$, then the relation

$$
y(z+L)=\sum_{j=0}^{L}\left(\begin{array}{l}
L \\
j
\end{array}\right) \Delta^{j} y(z), \quad L=0, \cdots, n,
$$

implies that we can transform (7.7) to (7.2). Then the corresponding $P_{0}$ obtained in (7.2) has the highest degree. That is, (7.1) holds. Therefore, the result of the corollary follows from Theorem 7.1 .

It is well known that each entire solution of the linear differential equation

$$
P_{n}(z) f^{(n)}(z)+\cdots+P_{1}(z) f^{\prime}(z)+P_{0}(z) f(z)=0,
$$

with polynomial coefficients, has order of growth equal to a rational number which can be determined from the gradients of the corresponding Newton-Puisseux diagram 25]. This classical result can be proved from the Wiman-Valiron theory 
(Lemmas 6.1 and 6.2) (see also [39, pp. 106-111, Appendix A] and [13]). We shall establish a corresponding result for the linear difference equation (7.2) but only for entire solutions with order strictly less than one. Our method is based on our "difference" Wiman-Valiron Theorem 6.1 Although the main idea of our argument of using (6.7) follows closely that of the classical one for linear differential equations, there are some details that require further justifications. The reason is due to the different forms of the remainders between (6.7) in our Theorem6.1] and the classical one in (6.3).

Theorem 7.3. Let $a_{0}(z), \cdots, a_{n}(z)$ be polynomial coefficients of the difference equation

$$
a_{n}(z) \Delta^{n} f(z)+\cdots+a_{1}(z) \Delta f(z)+a_{0}(z) f(z)=0 .
$$

Let $f$ be a transcendental entire solution of (7.10) with $\sigma(f)<1$. Then we have $\sigma(f)=\sigma=\chi$ where $\chi$ is a rational number which can be determined from a gradient of the corresponding Newton-Puisseux diagram of equation (7.10). In particular, $\chi>0$.

Proof. Let $f(z)$ be a transcendental entire solution of (7.10) with order $0<\sigma<1$. Lemma 6.1 asserts that

$$
\limsup _{r \rightarrow \infty} \frac{\log \nu(r, f)}{\log r}=\sigma .
$$

This implies that for each $\delta>0$, there exists a sequence $r_{n} \rightarrow \infty$, such that $r_{n+1}>r_{n}^{1+\delta}$ and $\nu\left(r_{n}, f\right) \geq r_{n}^{\sigma-\delta}$.

We define

$$
F=\bigcup_{n=1}^{\infty}\left[r_{n}, r_{n}^{1+\delta}\right]
$$

which is a union of non-intersecting intervals and clearly has infinite logarithmic measure. We consider all those $r$ that lie in

$$
r_{n} \leq r \leq r_{n}^{1+\delta} .
$$

Then since $\nu(r, f)$ is a non-decreasing function of $r$, so we have trivially

$$
\nu(r, f) \geq \nu\left(r_{n}, f\right) \geq r_{n}^{\sigma-\delta} \geq r^{\frac{\sigma-\delta}{1+\delta}} .
$$

We now choose $\delta$ and $\varepsilon$ so small that the inequality

$$
k \sigma-k-\gamma+\varepsilon<\left(\frac{\sigma-\delta}{1+\delta}-1\right) k
$$

holds, where $\gamma$ is the constant in (6.7). Hence

$$
r^{k \sigma-k-\gamma+\varepsilon}=o\left(\left|\frac{\nu(r, f)}{z}\right|^{k}\right),
$$

for $r$ in $F$. Let $z$ be such that (6.5) holds. Thus equation (6.7) of Theorem 6.1 asserts that there exists a set $E$ of finite logarithmic measure such that for all $|z|=r \in F \backslash\{E \cup[0,1]\}$, we have

$$
\frac{\Delta^{k} f(z)}{f(z)}=\eta^{k}\left(\frac{\nu(r, f)}{z}\right)^{k}(1+o(1)) .
$$


Substituting (7.17) into (7.10) yields

$$
\sum_{j=0}^{n} \eta^{j} a_{A_{j}}^{(j)} \nu^{j}(r, f) z^{A_{j}-j} f(z)(1+o(1))=0,
$$

where $A_{j}=\operatorname{deg} a_{j}(z), a_{j}(z)=\sum_{k=0}^{A_{j}} a_{k}^{(j)} z^{k}$.

Valiron [39, p. 108 and Appendix A] asserts that we have, for $r \in F \backslash\{E \cup[0,1]\}$, two real functions $B(r)$ and $\ell(r)$, where $\ell(r)$ can only take values equal to the gradients of the corresponding Newton-Puisseux diagram for equation (7.10) (which is always positive), and $B(r)$ also can only assume a finite number of positive values such that

$$
\lim _{r \rightarrow \infty} \frac{\nu(r, f)}{B(r) r^{\ell(r)}}=1
$$

In particular, since $\ell(r)$ can only take a finite number of rational values and $F \backslash\{E \cup$ $[0,1]\}$ has infinite logarithmic measure, so there exists a rational $\chi>0$ such that the set

$$
\{r: \ell(r)=\chi, \quad r \in F \backslash\{E \cup[0,1]\}\}
$$

is unbounded. We conclude from (7.14), (7.11), (7.19) and (7.20) that

$$
\frac{\sigma-\delta}{1+\delta} \leq \chi \leq \sigma .
$$

Hence $\sigma=\chi$ by letting $\delta \rightarrow 0$.

It only remains to consider the case when $\sigma=0$. Since the remainder of our "difference" Wiman-Valiron estimate (6.6) is the same as the classical one (6.3), so we simply repeat the classical argument as in [39. This also shows that $\sigma=0$ must be a gradient from the Newton-Puisseux diagram for equation (7.10), which is impossible.

We now consider a general first order algebraic difference equation. Pólya 31] proved the following classical theorem.

Theorem 7.4. Let $f(z)$ be an entire solution of the first order algebraic differential equation

$$
\Omega\left(z, f(z), f^{\prime}(z)\right)=0,
$$

with polynomial coefficients. Then $\sigma(f)>0$.

The proof utilizes the method of contradiction by assuming that if $f(z)$ has order zero, then the central index $\nu(r, f)$ satisfies

$$
\lim _{r \rightarrow \infty} \frac{\nu(r, f)^{k}}{r}=0
$$

for every positive integer $k$ and (6.3). Since our (6.6) for the difference operator resembles (6.3), so Pólya's argument applies verbatim to first order difference equations also with polynomial coefficients (see also [27, Chap. 11]). Thus we obtain

Theorem 7.5. Let $f(z)$ be an entire solution of the first order algebraic difference equation

$$
\Omega(z, f(z), \Delta f(z))=0,
$$

with polynomial coefficients. Then $\sigma(f)>0$. 


\section{CONCLUding REMARKS}

Our method of proof of the main theorems depends heavily on the Poisson-Jensen formula and is closely related to Nevanlinna theory. However, our results may be better understood via the following formal approach to the difference quotients and logarithmic derivatives. Let us denote by $E_{\eta}$ the operator $E_{\eta} f(z)=f(z+\eta)$, and define $D$ as $D f(z)=f^{\prime}(z)$. Then we may write $\Delta f(z)=\left(E_{\eta}-1\right) f(z)$. That is, $\Delta=E_{\eta}-1$ in the operator sense. Let us suppose in addition that we have the expansion

$$
\begin{aligned}
E_{\eta} f(z) & =f(z+\eta)=f(z)+\eta f^{\prime}(z)+\frac{\eta^{2}}{2 !} f^{\prime \prime}(z)+\cdots \\
& =f(z)+\eta D f(z)+\frac{\eta^{2}}{2 !} D^{2} f(z)+\cdots \\
& =\left(1+\eta D+\frac{\eta^{2} D^{2}}{2 !}+\frac{\eta^{3} D^{3}}{3 !}+\cdots\right) f(z) \\
& =\left(e^{\eta D}\right) f(z) .
\end{aligned}
$$

That is, we have formally $E_{\eta}=e^{\eta D}$ (see [28, p. 33]). Substituting $\Delta=E_{\eta}-1$ into it yields again formally

$$
\begin{aligned}
\Delta^{k} & =\left(e^{\eta D}-1\right)^{k} \\
& =\left(\eta D+\frac{\eta^{2} D^{2}}{2 !}+\frac{\eta^{3} D^{3}}{3 !}+\cdots\right)^{k} \\
& =\eta^{k} D^{k}+\frac{k}{2 !} \eta^{k+1} D^{k+1}+\cdots .
\end{aligned}
$$

Thus if we ignore the terms after the leading term in the last line in (8.2), which was vigorously justified in our argument in $\$ 3$, we obtain

$$
\frac{\Delta^{k} f}{f}=\eta^{k} \frac{f^{(k)}(z)}{f(z)}+(\text { small remainders })
$$

which is formally the same as in (1.5).

The basic relations (1.4) and (1.5) give rise to the different growth patterns of finite order meromorphic functions of order greater than one and of order strictly less than one respectively. While the relations in both categories deserve more detailed study, the relation (1.4) for functions with order exactly one appears to be the most difficult to handle. But this category of functions includes many important functions, such as many classical special functions from mathematical physics.

Methods for solving linear difference equations have been investigated by mathematicians as far back as the end of the nineteenth century. For example, MilneThomson [28, Chap. XIV] discussed methods for solving linear difference equations in terms of factorial series via the operators " $\pi$ " and " $\rho$ " that would "converge everywhere, or nowhere or converge in a half-plane in the right". It appears that the order estimates for the meromorphic solution of Theorem 7.1 and those for the entire solutions to the linear difference equations and first order non-linear difference equations in Theorem 7.3 and Theorem 7.5 respectively are new. The topics deserve further study. 


\section{ACKNOWLEDGEMENTS}

The authors would like to thank the referee for his/her constructive suggestions and for pointing out some errors in our original manuscript. These comments greatly improved the readability of the paper.

The second author thanks the Hong Kong University of Science and Technology for its hospitality during his visit from August 2004 to March 2005 where the main results presented in this paper were obtained.

\section{REFERENCES}

1. M. J. Ablowitz and P. A. Clarkson, Solitons, Nonlinear Evolution Equations and Inverse Scattering, London Math. Soc. Lect. Notes. Ser. No. 149, Cambridge Univ. Press, 1991. MR:1149378 (93g:35108)

2. M. J. Ablowitz, R. Halburd and B. Herbst, On the extension of the Painlevé property to difference equations, Nonlinearity 13 (2000), 889-905. MR.1759006 (2001g:39003)

3. W. Bergweiler and W. K. Hayman, Zeros of solutions of a functional equation, Comput. Methods Funct. Theory 3 (2003), no. 1-2, 55-78. MR2082005 (2005d:39088)

4. W. Bergweiler, K. Ishizaki and N. Yanagihara, Meromorphic solutions of some functional equations. Methods Appl. Anal. 5 (1998), no. 3, 248-258. MR1659151(2000a:39025)

5. W. Bergweiler, K. Ishizaki and N. Yanagihara, Growth of meromorphic solutions of some functional equations I, Aequationes Math., 63 (2002), 140-151. MR.1891282 (2003m:39036)

6. W. Bergweiler and J. K. Langley, Zeros of differences of meromorphic functions, Math. Proc. Cambridge Philos. Soc. 142 (2007) no. 1, 133-147. MR2296397 (2008e:30041)

7. H. Cartan, Sur les systèmes de fonctions holomorphes à variétés linéaires lacunaires et leurs applications, Ann. Sci. Ecole Norm. Sup., (3) 45 (1928), 255-346. MR1509288

8. Y. M. Chiang and S. J. Feng, On the Nevanlinna characteristic of $f(z+\eta)$ and difference equations in the complex plane, The Ramanujan J. 16 (2008), 105-129. MR.2407244

9. Y. M. Chiang and S. N. M. Ruijsenaars, On the Nevanlinna order of meromorphic solutions to linear analytic difference equations, Stud. Appl. Math., 116 (2006), 257-287. MR 2220338 (2006m:39005)

10. B. Grammaticos, A. Ramani and V. Papageorgiou, Do integrable mappings have the Painlevé property? Phys. Rev. Lett., 67 (1991), 1825-1828. MR1125950 (92f:58081)

11. V. I. Gromak, I. Laine, S. Shimomura, Painlevé Differential Equations in the Complex Plane, de Gruyter Studies in Math., Vol. 28, Walter de Gruyter, Berlin, 2002. MR1960811 (2003m:34210)

12. G. G. Gundersen, Estimates for the logarithmic derivative of meromorphic functions, plus similar estimates, J. London Math. Soc. (2) 37 (1988), 88-104. MR921748 (88m:30076)

13. G. G. Gundersen, E. M. Steinbart and S. P. Wang, The possible orders of solutions of linear differential equations with polynomial coefficients, Trans. Amer. Math. Soc., 350, no. 3, (1998), 1225-1247. MR1451603 (98h:34006)

14. R. G. Halburd and R. J. Korhonen, Difference analogue of the lemma on the logarithmic derivative with applications to difference equations, J. Math. Anal. Appl. 314 (2006), 477487. MR2185244 (2007e:39030)

15. R. G. Halburd and R. J. Korhonen, Finite-order meromorphic solutions and the discrete Painlevé equations, Proc. Lond. Math. Soc. (3) 94, (2007) 443-474. MR2308234(2008c:39006)

16. R. G. Halburd and R. J. Korhonen, Nevanlinna theory for the difference operator, Ann. Acad. Sci. Fenn. Math. 31 (2006) no. 2, 463-478. MR2248826 (2007e:30038)

17. R. G. Halburd and R.J. Korhonen, Existence of finite-order meromorphic solutions as a detector of integrability in difference equations, Phys. D. 218 (2006), 191-203. MR 2238752 (2007b:39032)

18. R. G. Halburd and R.J. Korhonen, Meromorphic solutions of difference equations, integrability and the discrete Painlevé equations, J. Phys. A: Math. Theor. 40 (2007), R1-R38. MR2343636

19. W. K. Hayman, Meromorphic Functions, Oxford Mathematical Monographs, The Clarendon Press, Oxford, 1964. MR0164038 (29:1337) 
20. W. K. Hayman, The local growth of power series: A survey of the Wiman-Valiron method, Canad. Math. Bull. 17 (1974), 317-358. MR0385095 (52:5965)

21. W. K. Hayman, On the zeros of a $q$-Bessel function, Contemporary Math., Vol. 382 (2005), 205-216. MR2175889 (2006m:30044)

22. Y. He and X. Xiao, Algebroid Functions and Ordinary Differential Equations, Beijing Sci. Press, 1988 (Chinese).

23. J. Heittokangas, R. Korhonen, I. Laine, J. Rieppo and K. Tohge, Complex difference equations of Malmquist type, Comput. Methods Funct. Theory, 1 (2001), 27-39. MR1931600 (2003i:39006)

24. J. Heittokangas, I. Laine, J. Rieppo and D. Yang, Meromorphic solutions of some linear functional equations, Aequationes Math., 60 (2000), 148-166. MR1777900 (2002f:39050)

25. E. Hille, Ordinary Differential Equations in the Complex Domain, Wiley, New York, 1976. MR.0499382 (58:17266)

26. K. Ishizaki and N. Yanagihara, Wiman-Valiron method for difference equations, Nagoya Math. J., 175 (2004), 75-102. MR2085312 (2006c:39001)

27. I. Laine, Nevanlinna Theory and Complex Differential Equations, Walter de Gruyter, Berlin 1993. MR 1207139 (94d:34008)

28. L. M. Milne-Thomson, The Calculus of Finite Differences, MacMillian \& Co., London, 1933.

29. B. Ja. Levin, Distribution of Zeros of Entire Functions, Vol. 5 Translations of Math. Mono., Amer. Math. Soc., 1980. MR589888 (81k:30011)

30. R. Nevanlinna, Analytic Functions, Springer-Verlag, New York, Heidelberg, Berlin, 1970. MR.0279280 (43:5003)

31. G. Pólya, Zur Untersuchung der Grössenordnung ganzer Funktionen, die einer Differentialgleichung genügen, Acta. Math. 42 (1920), 309-316, Jbuch 47, 410. MR.1555169

32. G. Pólya and G. Szegö, Problems and Theorems in Analysis, Vol. II, Springer-Verlag, Berlin, Heidelberg and New York, 1976. MR0396134 (53:2)

33. A. Ramani, B. Grammaticos and J. Hietarinta, Discrete versions of the Painlevé equations, Phys. Rev. Lett., 67 (1991) 1829-1832. MR1125951 (92j:39011)

34. A. Ramani, B. Grammaticos, T. Tamizhmani and K. M. Tamizhmani, The road to the discrete analogue of the Painlevé property: Nevanlinna meets singularity confinement, Comput. Math. Appl., 45 (2003), 1001-1012. MR2000573 (2005d:37147)

35. J.-P. Ramis, About the growth of entire function solutions of linear algebraic $q$-difference equations, Ann. Fac. Sci. Toulouse Math., (6) 1 (1992), no. 1, 53-94. MR1191729|(94g:39003)

36. S. N. M. Ruijsenaars, First order analytic difference equations and integrable quantum systems, J. Math. Phys., 40 (1997), 1069-1146. MR1434226 (98m:58065)

37. J. M. Whittaker, Interpolatory Function Theory, Cambridge Univ. Press, Cambridge, 1935.

38. E. T. Whittaker and G. N. Watson, A Course of Modern Analysis, $4^{\text {th }}$ edition, Cambridge Univ. Press, Cambridge, 1927. MR.1424469 (97k:01072)

39. G. Valiron, Lectures on the Theory of Integral Functions, Chelsea Publ. Co., 1949.

Department of Mathematics, Hong Kong University of Science and Technology, Clear Water Bay, Kowloon, Hong Kong, People's Republic of China

E-mail address: machiang@ust.hk

Academy of Mathematics and Systems Science, Chinese Academy of Sciences, Beijing, 100080, People's Republic of China

E-mail address: fsj@amss.ac.cn 